\title{
Perfil profesional docente y formación competitiva en egresados de una universidad de Ecuador
}

\section{Professional Teaching Profile and Competitive Training in Graduates of a University in Ecuador}

\author{
Wilson Eladio Tinoco-Izquierdo \\ Universidad Técnica de Machala, Machala, Ecuador \\ ORCID https://orcid.org/0000-0003-1162-6474 \\ Miguel Inga-Arias \\ Universidad Nacional Mayor de San Marcos, Lima, Perú \\ ORCID: https://orcid.org/0000-0002-1588-0181 \\ Jessica Paola Palacios-Garay \\ Universidad Nacional Mayor de San Marcos, Lima, Perú \\ ORCID: https://orcid.org/0000-0002-2315-1683

\section{Quiterio Trujillo Reyna} \\ Universidad Nacional Mayor de San Marcos, Lima, Perú \\ ORCID: https://orcid.org/0000-0001-5706-1005
}

Recibido 28-10-19 Revisado 02-12-19 Aprobado 02-01-20 En línea 28-01-20

\section{Correspondencia}

Email: wtinoco@utmachala.edu.ec

\section{Citar como:}

(C) Universidad San Ignacio de Loyola, Vicerrectorado de Investigación, 2020.

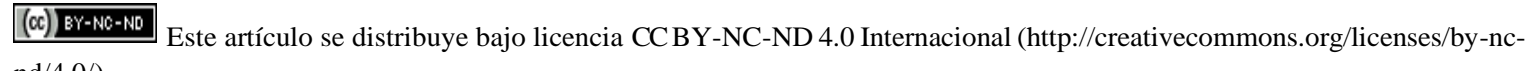
$\mathrm{nd} / 4.0 /)$.

Tinoco-Izquierdo, W., Inga-Arias, M., Palacios-Garay, J., \& Trujillo, Q. (2020). Perfil profesional docente y formación competitiva en egresados de una universidad de Ecuadorr. Propósitos y $\quad$ Representaciones,
http://dx.doi.org/10.20511/pyr2020.v8n3.443 


\section{Resumen}

La presente investigación busca demostrar los efectos que producen el perfil profesional del docente y su formación competitiva de los egresados de una universidad de Ecuador. El método de la investigación es transversal con un tipo de investigación aplicada cuantitativa, con una muestra de 55 docentes y 132 egresados. Se elaboraron y aplicaron dos encuestas para medir las variables; se usó el coeficiente de correlación de Pearson para determinar el grado y significancia de relación. Se concluye que: entre el perfil profesional del docente, la práctica docente, el desarrollo de funciones de investigación y vinculación con la colectividad, es $\mathrm{r}=0.82$. Entre el perfil profesional del docente, la práctica docente, el desarrollo de funciones de investigación y vinculación con la colectividad en la calidad de la formación profesional de los egresados, el coeficiente de Pearson es $\mathrm{r}=0.81, \mathrm{P}=0,00$ (sig. Bilateral) confirmándose que la relación entre las variables es significativa $\mathrm{p}<.05$.

Palabras clave: Perfil profesional; Práctica docente; Desarrollo docente; Investigación científica.

\section{Summary}

This research seeks to demonstrate the effects produced by the professional profile of the teacher and their competitive training of graduates of a university in Ecuador. The research method is transversal with a type of quantitative applied research, with a sample of 55 teachers and 132 graduates. Two surveys were developed and applied to measure the variables; Pearson's correlation coefficient was used to determine the degree and significance of relationship. It is concluded that: between the professional profile of the teacher, the teaching practice, the development of research functions and links with the community, it is $r=0.82$. Among the professional profile of the teacher, the teaching practice, the development of research functions and links with the community in the quality of the professional training of graduates, the Pearson coefficient is $\mathrm{r}=0.81, \mathrm{P}=0.00$ (sig. Bilateral) confirming that the relationship between the variables is significant $\mathrm{p}<.05$.

Keywords: Professional Profile; Teaching Practice; Teacher Development; Scientific Investigation.

\section{Introducción}

En esta última década en América Latina, la educación superior atraviesa por un proceso de optimización de la calidad (Escobar, Isabel, \& Guerra Bretaña, 2017; Gvirtz, \& Camou, 2018). Entre los factores que intervienen en la calidad educativa, uno de los de mayor trascendencia, es sin duda el perfil profesional del docente, dado que un adecuado perfil no solo garantiza el cumplimiento de los roles y funciones de sus áreas específicas de intervención, sino que además contribuye al mejoramiento de la producción investigativa, la vinculación con la colectividad y por ende en la competitividad institucional (Rangel Baca, 2015; Quilaqueo, Quintriqueo, \& Riquelme, 2016).

Una educación de calidad, es el producto o consecuencia de una observación crítica y sistematizada de los diferentes actores que participan en la práctica educativa y que conforman el colectivo institucional, especialmente por parte de los docentes. Una educación de calidad va más allá del objetivo de las instituciones educativas tradicionales, se trata de planear, ejecutar y liderar procesos educativos de cambio y transformación, donde los estudiantes sean cada vez más conscientes y responsables de sus capacidades, procesos y resultados de aprendizajes (Lugo \& Ithurburu, 2019). 
En consecuencia, para el colectivo institucional se presenta como elemento indispensable el contar con un perfil profesional idóneo que le permita diagnosticar, planear, ejecutar, innovar, solucionar, evaluar su tarea de manera más eficaz y eficiente con miras a la excelencia; contar con elementos que le ofrezca una visión más amplia e integradora de la problemática educativa y le permita una ruptura con la concepción tradicional de la educación fortaleciendo la práctica de la investigación y fomentar la vinculación con la colectividad Arango, Gutiérrez, Gavidia, \& Mora, 2015; Torres Carrillo, 2017). Actualmente la práctica educativa en Latinoamérica ha sido reducida a un conjunto de métodos, técnicas, recursos y estrategias metodológicas que únicamente fomentan la repetición acrítica, asistemática y poco reflexiva del estudiante, donde el educador conduce al educando en la memorización mecánica de los contenido (Marcelo, \& Vaillant, 2017; Tello, 2017).

El perfil de formación del docente universitario comprende no sólo el desarrollo de capacidades, competencias, habilidades y desempeños en su disciplina, sino, además, una sólida formación en las dimensiones personales, académicas, docentes e investigativas (Martínez-Chairez, Guevara-Araiza, \& Valles-Ornelas, 2016). De este modo, "la profesión docente está mutando y aumentando su complejidad (cambio del perfil discente, uso de nuevas metodologías orientadas a la adquisición de competencias, nuevas tecnologías como elemento transversal en la multivariedad de estrategias metodológicas que se le solicita que emplee, etc.) y por ello los requerimientos a este profesional son mayores, entre otros, en el domino de las competencias psicopedagógicas, tecnológicas, lingüísticas (dominio de una segunda y tercera lengua), etc.” (Gros y Romaña, 2004, pág. 148). Estos nuevos paradigmas han venido a transformar de fondo la forma en la que se enseñan conocimientos, cobrando ahora una mayor importancia, junto a los conocimientos, la forma en la que se aprende y se desarrollan valores, habilidades y actitudes, las cuales dependen de complejas interacciones sociales.

La investigación como base de la enseñanza y de formación permite al docente, desde la reflexión crítica, la construcción de saber dejando de lado el rol de mediador pasivo entre teoría y práctica (Caballero, \& Botía, 2015). Es deseable que cada profesor o grupo de profesores desarrolle una línea de investigación, con el fin de formalizar su trabajo y el de los colegas y en la medida de sus posibilidades y como resultado de trabajos de investigación generar patentes, proyectos para la industria, prototipos y demás, que de alguna manera proporcionen ingresos extraordinarios (Da Cunha, 2015).

Para Montes y Suárez, (2016) el nivel de calidad y productividad de los docentes en Instituciones de Educación Superior es un concepto que puede ser medido y evaluado de forma normativa o en términos relativos, esto no permiten una evaluación concreta y/o absoluta, lo que obliga a la creación de "indicadores" que permitan constituir un valioso instrumento que proporcione elementos de juicio para analizar a fondo los procesos educativos, convirtiéndose en un proceso que arroje información para promover y asegurar la mayor calidad y productividad de las acciones y resultados de la docencia.

Por lo anterior, es fundamental que los programas tengan procedimientos e instrumentos válidos y confiables para medir el logro de cada uno de los atributos del perfil de egreso; sin éstos es posible conocer el número de egresados, pero no si los egresados personifican el perfil de egreso. Los perfiles profesionales de egreso estarán constituidos por un conjunto de competencias cuyo trasfondo (y sentido terminal o producto) serían las capacidades y valores adquiridos por medio de contenidos y métodos al egresar de una institución educativa y válida para la vida y/o para una profesión. Teóricamente, la eficiencia de un programa de educación superior consiste en el logro del 
perfil del egresado. Se puede decir que la eficiencia terminal de un programa de educación superior es directamente proporcional al número de alumnos que alcanzan dicho perfil.

Lo mencionado anteriormente permite concluir que la práctica educativa en Latinoamérica ha sido reducida a un conjunto de métodos, técnicas, recursos y estrategias metodológicas que únicamente fomentan la repetición acrítica, asistemática y poco reflexiva del estudiante, donde el educador conduce al educando en la memorización mecánica de los contenidos. Por ello la presnet investigación nos permitirá responder la siguiente pregunta ¿Qué efectos producen el perfil profesional del docente, la práctica docente, el desarrollo de funciones de investigación y vinculación con la colectividad y su efecto en la calidad de la formación profesional de los egresados de una universidad de Ecuador?

\section{Método}

La investigación corresponde a un diseño no experimental transversal de tipo aplicada que pretende encontrar efectos producen el perfil profesional del docente, la práctica docente, el desarrollo de funciones de investigación y vinculación con la colectividad y su efecto en la calidad de la formación profesional de los egresados universitarios.

La muestra está conformada por 55 docentes y 132 Egresados de la Unidad Académica de Ciencias Sociales de una universidad de Ecuador.

\section{Instrumentos}

Se utilizaron fuente de datos primarios, según los planteado por Sabino 1992 como: "Aquellos que el investigador obtiene directamente de la realidad, recolectándolos con sus propios instrumentos" (p. 144). Fueron utilizados los procedimientos e instrumentos descritos a continuación:

La observación directa: Esta observación fue participativa, pues el investigador formó parte del grupo objeto de estudio como facilitador del plan de formación, a fin de determinar la problemática y dar soluciones. En sustento a la observación se realizaron anotaciones de cada una de las sesiones de trabajo las cuales se validaron a través de actas docentes.

La entrevista: se profundizó en los sentimientos, aptitudes, actitudes y apreciación de las autoridades en función de la formación que han tenido, la información que han buscado para conocer un poco más del tema y el impacto que ha tenido la formación en su crecimiento personal y profesional.

La encuesta: Se utilizó un cuestionario estructurado en 10 preguntas cerradas y una sección de comentarios y sugerencias, las cuales estuvieron redactadas de acuerdo a las variables establecidas.

\section{Resultados}

La matriz de correlación presenta por pares de variables el coeficiente de Pearson y el nivel de significancia obtenido, se tiene una significancia de 0.000 , menor a 0.05 , existiendo una relación significativa entre el perfil profesional del docente, y la formación competitiva profesional de los egresados de la unidad académica de ciencias sociales. Se encontró una asociación lineal estadísticamente significativa muy alta $(r \mathrm{P}=0.824, p<0.05)$ (ver tabla 1)

Propósitos y Representaciones 


\section{Tabla 1.}

Correlación entre el perfil profesional del docente y la formación competitiva profesional de los egresados

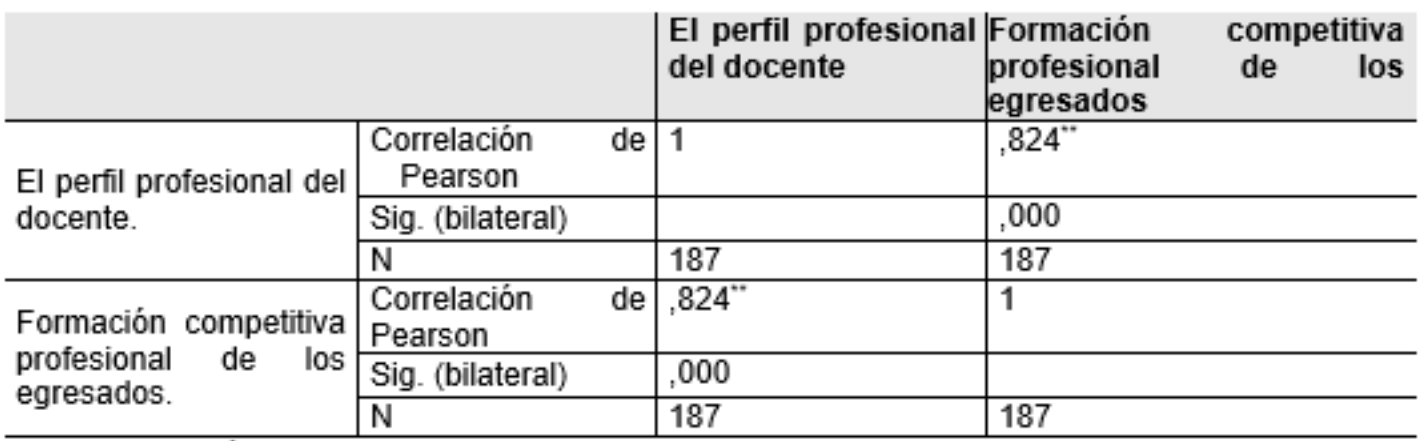

**. La correlación es significativa al nivel 0,01 (bilateral).

La matriz de correlación presenta por pares de variables el coeficiente de Pearson y el nivel de significancia obtenido, se tiene una significancia de 0.000 , menor a 0.05 , por lo que se rechaza la hipótesis nula, entonces: existe una relación significativa entre las dimensiones personales, las actividades pre-activas, el diseño, desarrollo y/o evaluación proyectos de investigación e innovación de relevancia para la docencia, el pedido de una organización o institución del medio y la formación competitiva profesional de los egresados. Se encontró una asociación lineal estadísticamente significativa moderada $(r \mathrm{P}=0.776, p<0.05)$ (ver tabla 2$)$

\section{Tabla 2.}

Correlación entre dimensiones personales, actividades pre-activas y otras con la formación competitiva profesional de los egresados

\begin{tabular}{l|l|l|l}
\hline \multicolumn{2}{l|}{} & $\begin{array}{l}\text { Las dimensiones } \\
\text { personales, } \\
\text { actividades pre- } \\
\text { activas y otras }\end{array}$ & $\begin{array}{l}\text { Formación } \\
\text { competitiva do los } \\
\text { profesional de los } \\
\text { egresados }\end{array}$ \\
\hline \multirow{2}{*}{$\begin{array}{l}\text { Las dimensiones } \\
\text { personales, actividades } \\
\text { pre-activas y otras }\end{array}$} & $\begin{array}{l}\text { Correlación } \\
\text { Pearson }\end{array}$ & 1 &, $816^{* *}$ \\
\cline { 2 - 4 } & Sig. (bilateral) & &, 000 \\
\hline \multirow{2}{*}{$\begin{array}{l}\text { Formación competitva } \\
\text { profesional de los } \\
\text { egresados }\end{array}$} & $\begin{array}{l}\text { Correlación de } \\
\text { Pearson }\end{array}$ & 187 & 187 \\
\cline { 2 - 4 } & Sig. (bilateral) &, $816^{* *}$ & 1 \\
\cline { 2 - 5 } & $\mathrm{N}$ & 187 & 187 \\
\hline
\end{tabular}

**. La correlación es significativa al nivel 0,01 (bilateral).

La matriz de correlación presenta por pares de variables el coeficiente de Pearson y el nivel de significancia obtenido, se tiene una significancia de 0.000 , menor a 0.05 , entonces: existe una relación significativa entre las dimensiones académicas, las actividades interactivas, la organización y gestión de reuniones científicas, el interés del docente y/o estudiantes en la realización del proyecto y la formación competitiva profesional de los egresados de la unidad académica de ciencias sociales, 
Se encontró una asociación lineal estadísticamente significativa muy alta ( $r \mathrm{P}=0.827, p<0.05$ ) (ver tabla 3)

\section{Tabla 3.}

Matriz de correlación entre las dimensiones académicas, las actividades interactivas y otras con la formación competitiva profesional de los egresados.

\begin{tabular}{|c|c|c|c|}
\hline & & $\begin{array}{l}\text { las dimensiones } \\
\text { académicas, las } \\
\text { actividades } \\
\text { interactivas, }\end{array}$ & \begin{tabular}{|l} 
Formación \\
competitiva \\
profesional de los \\
egresados \\
\end{tabular} \\
\hline \multirow{3}{*}{$\begin{array}{l}\text { Las dimensiones } \\
\text { académicas, las } \\
\text { actividades } \\
\text { interactivas, }\end{array}$} & $\begin{array}{l}\text { Correlación de } \\
\text { Pearson }\end{array}$ & 1 & $827^{*+}$ \\
\hline & Sig. (bilateral) & &, 000 \\
\hline & $\mathrm{N}$ & 187 & 187 \\
\hline \multirow{3}{*}{$\begin{array}{lcc}\text { Formación } & \text { competitva } \\
\text { profesional } & \text { de } & \text { los } \\
\text { egresados } & & \end{array}$} & $\begin{array}{l}\text { Correlación de } \\
\text { Pearson }\end{array}$ &, $827^{+*}$ & 1 \\
\hline & Sig. (bilateral) & .000 & \\
\hline & $\mathrm{N}$ & 187 & 187 \\
\hline
\end{tabular}

**. La correlación es significativa al nivel 0,01 (bilateral).

La matriz de correlación presenta por pares de variables el coeficiente de Pearson y el nivel de significancia obtenido, se tiene una significancia de 0.000 , menor a 0.05 , entonces: existe una relación significativa entre los docentes y las actividades post-activas, la elaboración de material científico actual y relevante para la docencia, las necesidades detectadas por la interacción entre la Universidad y los distintos actores sociales involucrados y la formación competitiva profesional de los egresados de la unidad académica de ciencias sociales. Se encontró una asociación lineal estadísticamente significativa muy alta $(r \mathrm{P}=0.836, p<0.05)$ (ver tabla 4$)$ 


\section{Tabla 4.}

Correlación entre la dimensión los docentes y las actividades post-activas y otros con la variable calidad de la formación profesional de los egresados

\begin{tabular}{|c|c|c|c|}
\hline & & $\mid \begin{array}{l}\text { Los docentes y las } \\
\text { actividades post-activas } \\
\text { y otros }\end{array}$ & \begin{tabular}{|l} 
Formación \\
competititva \\
profesional de \\
los egresados \\
\end{tabular} \\
\hline \multirow{3}{*}{$\begin{array}{l}\text { Los docentes y las } \\
\text { actividades post- } \\
\text { activas y otras }\end{array}$} & Correlación de Pearson & 1 &, $836^{*}$ \\
\hline & Sig. (bilateral) & &, 000 \\
\hline & $\mathrm{N}$ & 187 & 187 \\
\hline \multirow{3}{*}{$\begin{array}{l}\text { Formación } \\
\text { competitva } \\
\text { profesional de los } \\
\text { egresados }\end{array}$} & Correlación de Pearson &, $836^{*+}$ & 1 \\
\hline & Sig. (bilateral) &, 000 & \\
\hline & $\mathrm{N}$ & 187 & 187 \\
\hline
\end{tabular}

**. La correlación es significativa al nivel 0,01 (bilateral).

matriz de correlación presenta por pares de variables el coeficiente de Pearson y el nivel de significancia obtenido, se tiene una significancia de 0.000 , menor a 0.05 , por lo que se rechaza la hipótesis nula, entonces: existe una relación significativa entre las dimensiones investigativas la Comunicación y difusión conocimientos, avances científicos, resultados de proyectos de investigación e innovación a nivel nacional e internacional y calidad formación competitiva profesional de los egresados. Se encontró una asociación lineal estadísticamente significativa muy alta $(r \mathrm{P}=0.803, p<0.05)$ (ver tabla 5).

\section{Tabla 5.}

Correlación entre las dimensiones investigativas, la Comunicación y difusión conocimientos, avances científicos con la formación competitva profesional de los egresados

\begin{tabular}{|c|c|c|c|}
\hline & & $\begin{array}{l}\text { Dimensiones investigativas } \\
\text { la Comunicación y difusión } \\
\text { conocimientos, avances } \\
\text { científicos. }\end{array}$ & $\begin{array}{l}\text { Formación competitiva } \\
\text { profesional de los } \\
\text { egresados }\end{array}$ \\
\hline \multirow{3}{*}{$\begin{array}{l}\text { Dimensiones investigativas } \\
\text { la Comunicación y difusión } \\
\text { conocimientos, avances } \\
\text { científicos, }\end{array}$} & $\begin{array}{l}\text { Correlación de } \\
\text { Pearson }\end{array}$ & 1 & $805^{\prime \prime}$ \\
\hline & Sig. (bilateral) & &, 000 \\
\hline & $\mathrm{N}$ & 187 & 187 \\
\hline \multirow{3}{*}{$\begin{array}{l}\text { Formación competitiva } \\
\text { profesional de los egresados }\end{array}$} & $\begin{array}{l}\text { Correlación de } \\
\text { Pearson }\end{array}$ & $805^{\prime \prime}$ & 1 \\
\hline & Sig. (bilateral) &, 000 & \\
\hline & $\mathrm{N}$ & 187 & 187 \\
\hline
\end{tabular}

\section{Resultados}

Los resultados obtenidos en la dimensión Formación Profesional Recibida, Relación del perfil de ingreso y egreso, Organización estructural, Resultados del proceso de formación, Infraestructura, Vinculación con el medio, Satisfacción en la formación recibida, se encontró una significancia 
bilateral menor al alfa $(0,05)$ por lo que se deduce que existe efecto significativa con una correlación de ,824 en ese sentido estudios realizados por Rodríguez (2016) señala que la formación de los egresados ha sufrido un estancamiento en el ámbito laboral, condición que muestra el desarrollo de las profesiones, el estancamiento en el progreso de los ingresos percibidos muestra de forma muy significativa la pérdida del poder adquisitivo de los salarios percibidos por diferentes profesiones. Por su parte De Gasperin; Pérez; Gutiérrez; Salazar. (2016) señalan que el desempeño profesional de los egresados, en lo que se refiere a las competencias profesionales y las genéricas, así como las carencias en la formación profesional para desempeñarse en el mundo laboral actual, los egresados de un Sistema Abierto tienen algunas características especiales con respecto a egresados de un sistema convencional.

En esta investigación se corroboró la dimensión Diseño, desarrollo y/o evaluación proyectos de investigación e innovación de relevancia para la docencia encontrándose que causan efectos significativos con una significancia de ,816. En la dimensión elaboración de material científico actual y relevante para la docencia los resultados al que se llegaron en la investigación fue que existe una significancia bilateral de 0.00 . menor al alfa $(0,05)$ por lo que se afirma que el desarrollo de funciones de investigación; sin embargo estudios realizados por Mas (2011) manifiesta que la universidad, es el lugar donde el profesor universitario desarrolla su trabajo, está en uno de los mayores momentos de transformación de su historia; algunos de estos procesos han sido provocados directamente por los cambios sociales que se están sucediendo, otros por la tendencia al "rendimiento de cuentas" que se está instaurando en el sector público y otros, concretamente, por la convergencia hacia un Espacio Europeo de Educación (EEES).

Asimismo, Alfaro \& Alvarado (2016) en sus resultados muestran la relevancia en cuestiones vinculadas con el conocimiento y con estrategias didácticas básicas con las que debe contar el docente. La percepción del alumnado masculino y femenino no difiere demasiado en cuestiones básicas y consideradas prioritarias para elaborar el perfil del buen docente universitario. La cual se asemeja con una de las dimensiones estudiadas en el presente estudio. En tanto, Battauz (2014) en sus resultados señala que es importante destacar que ingresaron por concurso de antecedentes y cuando se indaga sobre su grado de satisfacción con el empleo en un porcentaje mayor respondieron satisfactoriamente y muy satisfactoriamente, no se registraron respuestas poco satisfactorias o insatisfactorias. En cuanto a la formación académica recibida respondieron, en términos generales, que es satisfactoria y luego muy satisfactoria, no registrándose ninguna respuesta poco satisfactoria o insatisfactoria. La cual tiene similitud con lo investigado en los indicadores de las variables de estudio.

Finalmente se comprobó si lo que plantea Romero \& Rodríguez en cuanto a la satisfacción laboral está en función de que las necesidades de remuneración, afiliación, logro y autorrealización sean cubiertas y se manifiesta en la actitud de los empleados hacia el trabajo, ambiente de trabajo y rendimiento. En la que los resultados muestran que la mayor satisfacción laboral se centra en el ambiente de trabajo, la posibilidad de hacer algo para la sociedad y el trabajo en equipo. Por el contrario, la insatisfacción se relaciona con el salario percibido y los esquemas tradicionales empresariales. Estos resultados se asemejan a los resultados presentados en los resultados del presente estudio.

Lo anterior nos permite recomendar la implementación de cursos, seminarios, capacitaciones que permitan al egresado experimentar fuera de las aulas universitarias y realice lo que aprendió en el transcurso de los años estudiantiles para que se pueda desenvolver socialmente. Además de ampliar las competencias investigativas de los estudiantes para lograr un interés real ya que es una realidad que el verdadero profesional debe demostrar en la sociedad y pueda realizar cambios con las 
investigaciones sociales y lograr una mejora en la calidad educativa. Se propone en elaborar una propuesta de un plan integral de formación docente para mantener la excelencia y la pertinencia educativa, en vista que la Educación a Distancia y las Tecnologías de Información y Comunicación componen en la actualidad un binomio imprescindible, debido a que estas tecnologías han posibilitado la aparición de espacios educativos virtuales que ofrecen nuevas y diversas vías hacia el aprendizaje representadas por su flexibilidad.

\section{Reeferencias}

Alfaro, G. \& Alvarado S. (2016). El Perfil de profesores universitarios de universidades públicas y privadas en la carrera de Educación. Consejo Nacional de Enseñanza Superior Privada (CONESUP), Ministerio de Educación Pública, Instituto de Investigación en Educación (INIE), Costa Rica.

Battauz, Liliana (2014). La inserción laboral y la formación profesional de los egresados en bibliotecología de la Facultad de Ciencias de la Gestión - UADER -Seguimiento correspondiente al período 2009-2012. Universidad Nacional del Litoral, Santa Fe Argentina.

Arango, M. I. A., Gutiérrez, R., Gavidia, V., \& Mora, R. M. (2015). Representaciones mentales colectivas sobre salud en el currículo de una Institución Educativa Distrital de la ciudad de Barranquilla. Educación y Humanismo, 17(28), 89-101.

Gvirtz, S., \& Camou, A. (2018). La universidad argentina en discusión: sistemas de ingreso, financiamiento, evaluación de la calidad y relación universidad-Estado. Ediciones Granica.

Caballero, K., \& Botía, A. B. (2015). El profesorado univeristario como docente: hacia una identidad profesional que integre docencia e investigación. REDU: Revista de Docencia Universitaria, 13(1), 4.

Da Cunha, M. I. (2015). Investigación y docencia: escenarios y senderos epistemológicos para la evaluación de la educación superior. REDU: Revista de Docencia Universitaria, 13(1), 5.

Escobar, J., Isabel, A., \& Guerra Bretaña, R. M. (2017). El liderazgo y la participación como factores clave para la gestión de la calidad. Caso de la Universidad Estatal de Bolívar. Cofin Habana, 11(2), 206-225.

Lugo, M. T., \& Ithurburu, V. (2019). Políticas digitales en América Latina. Tecnologías para fortalecer la educación de calidad. Revista Iberoamericana de Educación, 79(1), 11-31.

Marcelo, C., \& Vaillant, D. (2017). Políticas y programas de inducción en la docencia en Latinoamérica. Cadernos de Pesquisa, 47(166), 1224-1249.

Martínez-Chairez, G. I., Guevara-Araiza, A., \& Valles-Ornelas, M. M. (2016). El desempeño docente y la calidad educativa. Ra Ximhai, 12(6), 123-134.

Montes, D. A., \& Suárez, C. I. (2016). La formación docente universitaria: claves formativas de universidades españolas. Revista electrónica de investigación educativa, 18(3), 51-64.

Rangel Baca, A. (2015). Competencias docentes digitales: propuesta de un perfil. Píxel-Bit. Revista de Medios y Educación, 46, 235-248.

Quilaqueo, D., Quintriqueo, S., \& Riquelme, E. (2016). Identidad profesional docente: Práctica pedagógica en contexto Mapuche. Estudios pedagógicos (Valdivia), 42(2), 269-284.

Tello, C. (2017). Políticas educativas en Latinoamérica: la vinculación entre los investigadores académicos y tomadores de decisiones en educación. Un análisis desde la teoría de los campos. universitas humanística, (83), 57-82.

Torres Carrillo, A. (2017). Prácticas educativas en movimientos sociales de América Latina. Folios, (46), 3-14. 
Rodríguez P., (2016) Formación profesional de los egresados y su inserción en el mercado laboral”. Universidad Autónoma del Estado de México Revista Electrónica sobre Tecnología, Educación y Sociedad, 4(7).

De Gasperin S. Roberto; Pérez C. Nydia; Gutiérrez H. Luz María; Salazar R. Elsa A. (2016) El desempeño profesional de los egresados del Sistema Abierto de la Universidad Veracruzana desde la perspectiva de los empleadores. Universidad de Guadalajara.

Romero L., Martínez, Y., \& Rodríguez, L. (2014). Satisfacción laboral: estudio exploratorio en egresados la Universidad Autónoma de Sinaloa. Universidad Autónoma de Sinaloa- México. 